\title{
Trilinear-augmented gaugino mediation
}

\author{
Jan Heisig, ${ }^{a}$ Jörn Kersten, ${ }^{b}$ Nick Murphy ${ }^{c}$ and Inga Strümke ${ }^{b}$ \\ ${ }^{a}$ Institute for Theoretical Particle Physics and Cosmology, RWTH Aachen University, \\ 52056 Aachen, Germany \\ ${ }^{b}$ Department of Physics and Technology, University of Bergen, \\ 5020 Bergen, Norway \\ ${ }^{c} C P^{3}$-Origins, University of Southern Denmark, \\ 5230 Odense $M$, Denmark \\ E-mail: heisig@physik.rwth-aachen.de, joern.kersten@uib.no, \\ murphy@cp3.sdu.dk, inga.strumke@uib.no
}

ABSTRACT: We consider a gaugino-mediated supersymmetry breaking scenario where in addition to the gauginos the Higgs fields couple directly to the field that breaks supersymmetry. This yields non-vanishing trilinear scalar couplings in general, which can lead to large mixing in the stop sector providing a sufficiently large Higgs mass. Using the most recent release of FEynHiggs, we show the implications on the parameter space. Assuming a gravitino LSP, we find allowed points with a neutralino, sneutrino or stau NLSP. We test these points against the results of Run 1 of the LHC, considering in particular searches for heavy stable charged particles.

Keywords: Supersymmetry Phenomenology

ArXiv EPRINT: 1701.02313 


\section{Contents}

1 Introduction 1

2 Gaugino-mediated supersymmetry breaking 2

2.1 General setup 2

2.2 Trilinear couplings 2

2.3 Constraints from naïve dimensional analysis 4

3 Phenomenology of the model 5

3.1 Higgs mass 6

3.2 Particle spectrum $\quad 8$

$\begin{array}{lll}3.3 & \text { Tests at colliders } & 10\end{array}$

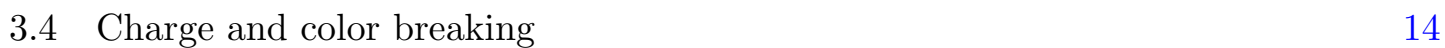

$\begin{array}{ll}3.5 \text { Cosmological constraints } & 15\end{array}$

4 Conclusions $\quad 15$

\section{Introduction}

Gaugino mediation $[1,2]$ is a mechanism for mediating supersymmetry breaking in a setup with extra spacetime dimensions, which avoids flavor problems by suppressing the soft sfermion masses at a high-energy scale. The original version of the model also yields suppressed trilinear scalar couplings, which is unfortunate since the measured Higgs mass [3] then requires a unified gaugino mass of $m_{1 / 2} \gtrsim 3 \mathrm{TeV}$ and thus very heavy sparticles [4].

However, a simple extension of the scenario does allow for non-vanishing trilinears and thus a lighter sparticle spectrum [5]. The couplings arise proportional to Yukawa couplings and thus do not lead to problematic flavor violation. We will investigate this possibility in detail in section 2, demonstrating explicitly how the trilinear couplings can be obtained.

In section 3, we study the parameter space of the extended setup. We show that the non-zero trilinears make it possible to reach the observed Higgs mass with sparticle masses that are accessible at the LHC. In gaugino mediation the gravitino can be the lightest supersymmetric particle (LSP) [6], making it a viable dark matter candidate [7]. ${ }^{1}$ We assume this scenario, in which case the next-to-lightest sparticle (NLSP) can be a stau, a tau sneutrino or a neutralino [8]. We determine the corresponding parts of the parameter space and constrain them by a careful analysis of LHC searches using data of the complete Run 1, in particular searches for long-lived heavy charged particles, extending the analysis in [5].

\footnotetext{
${ }^{1}$ Alternatively, another superweakly interacting particle such as the axino could be the LSP.
} 


\section{Gaugino-mediated supersymmetry breaking}

\subsection{General setup}

The present work considers one out of a class of higher-dimensional models. There are in general $D$ spacetime dimensions, $D-4$ of which are compact with volume $V_{D-4}$. This size determines the energy scale $M_{c} \equiv\left(1 / V_{D-4}\right)^{\frac{1}{D-4}}$ needed to resolve the compact dimensions, referred to as the compactification scale. Fields can either live in the whole $D$-dimensional space referred to as the bulk or be localized on $3+1$-dimensional branes that are located at different positions in the extra dimensions. The $D$-dimensional Lagrangian is [9]

$$
\mathcal{L}_{D}=\mathcal{L}_{\text {bulk }}(\hat{\Phi}(x, y))+\sum_{j} \delta^{(D-4)}\left(y-y_{j}\right) \mathcal{L}_{j}\left(\hat{\Phi}\left(x, y_{j}\right), \phi_{j}(x)\right),
$$

where $j$ runs over the branes, $x$ are coordinates on the branes, $y$ are coordinates in the bulk, $\hat{\Phi}$ is a bulk field ${ }^{2}$ and $\phi_{j}$ is a field localized on the $j$ th brane. Hats denote bulk fields with canonically normalized kinetic terms in $D$ dimensions.

We consider a model with two branes: the MSSM brane, where the visible matter fields are localized, and the hidden brane with a chiral superfield $S$, which is a singlet under the Standard Model (SM) gauge groups. Supersymmetry (SUSY) is broken by the vacuum expectation value (VEV) $\left\langle F_{S}\right\rangle$ of the auxiliary field of $S$. The gauge and Higgs superfields propagate in the bulk. Therefore, they can couple directly to the SUSY-breaking field and obtain soft masses proportional to $\left\langle F_{S}\right\rangle$. In contrast, sfermion soft masses are strongly suppressed due to the separation between the MSSM and hidden brane, which avoids unacceptably large flavor-changing neutral currents $[1,2]$.

\subsection{Trilinear couplings}

The supersymmetric part of the MSSM Lagrangian contains both bulk fields and fields constrained to the visible brane,

$$
\begin{aligned}
\mathcal{L}_{\mathrm{MSSM}} & =\mathcal{L}_{\text {bulk }}+\delta^{(D-4)}\left(y-y_{1}\right) \mathcal{L}_{1} \\
& =\left[W\left(\hat{\Phi}, \phi_{1}\right)+\frac{1}{4} \hat{\mathcal{W}}_{\alpha} \hat{\mathcal{W}}^{\alpha}\right]_{F}+\text { h.c. }+\left[K\left(\hat{\Phi}, \hat{\Phi}^{\dagger}, \phi_{1}, \phi_{1}^{\dagger}, e^{V}\right)\right]_{D},
\end{aligned}
$$

where $W$ is the visible-sector superpotential, $\hat{\mathcal{W}}$ the field strength superfield and $K$ the Kähler potential. Using the notation of equation (2.1), we have $j=1$ for the visible brane and will accordingly use $j=2$ for the hidden brane. On this brane, the gauge and Higgs superfields interact with the hidden-sector field $S$,

$$
\begin{aligned}
\mathcal{L}_{2}= & \frac{1}{M^{D-3}}\left[\frac{h}{4} S \hat{\mathcal{W}}^{\alpha} \hat{\mathcal{W}}_{\alpha}\right]_{F}+\text { h.c. } \\
& +\frac{1}{M^{D-3}}\left[S\left(a \hat{H}_{u}^{\dagger} \hat{H}_{d}^{\dagger}+b_{u} \hat{H}_{u}^{\dagger} \hat{H}_{u}+b_{d} \hat{H}_{d}^{\dagger} \hat{H}_{d}\right)+\text { h.c. }\right]_{D} \\
& +\frac{1}{M^{D-2}}\left[S^{\dagger} S\left(c_{u} \hat{H}_{u}^{\dagger} \hat{H}_{u}+c_{d} \hat{H}_{d}^{\dagger} \hat{H}_{d}+\left(d \hat{H}_{u} \hat{H}_{d}+\text { h.c. }\right)\right)\right]_{D}+\ldots
\end{aligned}
$$

\footnotetext{
${ }^{2}$ Strictly speaking, we use superfields of $4 \mathrm{D} N=1$ supersymmetry. The higher-dimensional supersymmetry requires additional fields, which we do not write explicitly, since they are not relevant here.
} 
where $h, a, b_{u, d}, c_{u, d}$ and $d$ are dimensionless couplings. The dots refer to terms containing only hidden-sector fields. Setting $b_{u, d}=0$ reduces the present case to the one considered in [2]. Setting also $a=c_{u, d}=d=0$, i.e., not placing the Higgs fields in the bulk, reduces our case to the one in [1]. Note that the localizations of $S$ and the sfermions forbid terms like $S \bar{u} \hat{H}_{u} Q$ and $S Q^{\dagger} Q$, which would directly yield trilinear couplings and sfermion soft masses.

Interactions between the bulk fields and the hidden-sector field are non-renormalizable, so $\mathcal{L}_{D}$ describes an effective theory valid up to some fundamental scale $M$. To obtain the 4-dimensional effective theory valid below the compactification scale, we integrate over the extra dimensions and keep only the zero modes of the bulk fields, which are constant in the extra dimensions. The integration yields a volume factor $V_{D-4}$ in the kinetic terms of the bulk fields, so we define fields with canonical kinetic terms in $4 \mathrm{D}$ by $\Phi \equiv \sqrt{V_{D-4}} \hat{\Phi}$. Thus, the part of the effective 4D Lagrangian describing the interactions of $S$ with the visible sector is

$$
\begin{aligned}
\mathcal{L}_{D=4} \supset & \frac{1}{V_{D-4}}\left\{\frac{1}{M^{D-3}}\left[\frac{h}{4} S \mathcal{W}^{\alpha} \mathcal{W}_{\alpha}\right]_{F}+\right.\text { h.c. } \\
& +\frac{1}{M^{D-3}}\left[S\left(a H_{u}^{\dagger} H_{d}^{\dagger}+b_{u} H_{u}^{\dagger} H_{u}+b_{d} H_{d}^{\dagger} H_{d}\right)+\text { h.c. }\right]_{D} \\
& \left.+\frac{1}{M^{D-2}}\left[S^{\dagger} S\left(c_{u} H_{u}^{\dagger} H_{u}+c_{d} H_{d}^{\dagger} H_{d}+\left(d H_{u} H_{d}+\text { h.c. }\right)\right)\right]_{D}\right\} .
\end{aligned}
$$

The first term generates gaugino masses $[1,2]$. We assume a unified gauge theory above the compactification scale, so that there is a unified gaugino mass $m_{1 / 2}$. The remaining terms produce the $B \mu$-term, soft Higgs masses $m_{H_{u}}^{2}$ and $m_{H_{d}}^{2}$, and a contribution to the $\mu$-term [2].

The terms proportional to $b_{u}$ and $b_{d}$, which were not included in the original versions of gaugino mediation [1, 2], contribute to the soft Higgs masses and $B \mu$ as well. Most importantly, however, they yield trilinear scalar couplings [5]. This can be seen by absorbing them via the field redefinitions $H_{u, d}^{\prime} \equiv H_{u, d}\left(1+b_{u, d} \frac{S}{M}\right)$, from the general expressions for soft SUSY-breaking terms in the supergravity formalism, see e.g. [10,11], or by integrating out the Higgs auxiliary fields. We find it instructive to show the latter calculation for our particular case.

First, the part of the Lagrangian (2.2) that contains the Higgs supermultiplets' auxiliary fields $F_{H_{u, d}}$ is

$$
\begin{aligned}
\mathcal{L}_{\mathrm{MSSM}} \supset F_{H_{u}}^{\dagger} F_{H_{u}}+F_{H_{d}}^{\dagger} F_{H_{d}}+\left(\phi_{\bar{u}} y_{u} F_{H_{u}} \phi_{Q}-\right. & \phi_{\bar{d}} y_{d} F_{H_{d}} \phi_{Q}-\phi_{\bar{e}} y_{e} F_{H_{d}} \phi_{L} \\
& \left.+\mu F_{H_{u}} \phi_{H_{d}}+\mu \phi_{H_{u}} F_{H_{d}}+\text { h.c. }\right),
\end{aligned}
$$

where $\phi_{X}$ denotes the scalar component of the superfield $X$. Adding the $D$-terms from equation (2.4) and employing the equations of motion $\partial \mathcal{L} / \partial F_{H_{u, d}}^{\dagger}=0$ yields

$$
F_{H_{u, d}}=-\frac{1}{V_{D-4} M^{D-3}}\left(b_{u, d} F_{S} \phi_{H_{u, d}}+b_{u, d} \phi_{S} F_{H_{u, d}}+b_{u, d}^{*} \phi_{S}^{*} F_{H_{u, d}}\right)+\ldots
$$

where we have omitted terms that do not contribute to SUSY-breaking trilinears. ${ }^{3}$ The

\footnotetext{
${ }^{3}$ Note that the term proportional to $a$ contributes to the supersymmetric (scalar) ${ }^{3}$ couplings. If the scalar component of $S$ develops a VEV, the terms proportional to $c_{u, d}$ also contribute to the trilinears, but this contribution can be absorbed by a redefinition of $b_{u, d}$.
} 
solutions are thus

$$
F_{H_{u, d}}=-\frac{\frac{b_{u, d} F_{S} \phi_{H_{u, d}}}{V_{D-4} M^{D-3}}}{1+b_{u, d} \frac{\phi_{S}}{V_{D-4} M^{D-3}}+b_{u, d}^{*} \frac{\phi_{S}^{*}}{V_{D-4} M^{D-3}}}+\cdots=-b_{u, d}\left(\frac{M_{c}}{M}\right)^{D-4} \frac{F_{S}}{M} \phi_{H_{u, d}}+\ldots
$$

omitting irrelevant higher-order terms in $\phi_{S}$ and replacing the extra dimensions' volume by the compactification scale in the last step. Substituting $F_{H_{u}}$ and $F_{H_{d}}$ into the Lagrangian (2.5) and replacing $F_{S}$ by its VEV finally gives rise to the desired trilinear terms,

$$
\mathcal{L}_{\text {trilinear }}=\left(\frac{M_{c}}{M}\right)^{D-4} \frac{\left\langle F_{S}\right\rangle}{M}\left(-b_{u} \phi_{\bar{u}} y_{u} \phi_{H_{u}} \phi_{Q}+b_{d} \phi_{\bar{d}} y_{d} \phi_{H_{d}} \phi_{Q}+b_{d} \phi_{\bar{e}} y_{e} \phi_{H_{d}} \phi_{L}+\text { h.c. }\right) \text {. }
$$

Consequently, we obtain trilinear scalar couplings proportional to the SUSY-breaking VEV and the Yukawa matrices,

$$
a_{u}=A_{u 0} y_{u} \quad, \quad a_{d}=A_{d 0} y_{d} \quad, \quad a_{e}=A_{d 0} y_{e}
$$

with

$$
A_{u 0}=\left(\frac{M_{c}}{M}\right)^{D-4} \frac{\left\langle F_{S}\right\rangle}{M} b_{u} \quad, \quad A_{d 0}=\left(\frac{M_{c}}{M}\right)^{D-4} \frac{\left\langle F_{S}\right\rangle}{M} b_{d}
$$

Due to the proportionality of trilinear matrices and Yukawa matrices in the relations (2.9), these matrices are simultaneously diagonalized when changing to the superCKM basis. Although the running to low energies leads to deviations from the exact proportionality, they are small enough to suppress flavor-changing neutral currents below the experimental upper limits.

Interestingly, the proportionality factors $A_{u 0}$ for the up-type squarks and $A_{d 0}$ for the down-type squarks and charged sleptons are different in general, in contrast to other simple setups for SUSY breaking like the Constrained MSSM or non-universal Higgs mass (NUHM) scenarios [12]. In the following we will restrict ourselves to the simplest possibility $A_{u 0}=A_{d 0} \equiv A_{0}$.

\subsection{Constraints from naïve dimensional analysis}

We will now estimate an upper limit on the trilinears, arguing that the couplings between the hidden-sector brane field $S$ and the bulk fields can be constrained by naïve dimensional analysis (NDA) [9]. This discussion generalizes results of [8], where the specific case of a 6-dimensional model was considered, to an arbitrary number of dimensions.

We write the Lagrangian (2.4) in terms of dimensionless fields $\breve{H}_{u, d}$ and $\breve{S}$ defined by

$$
H_{u, d}=\left(\frac{M^{D-2} V_{D-4}}{l_{D} / C}\right)^{1 / 2} \breve{H}_{u, d} \quad, \quad S=\left(\frac{M^{2}}{l_{4} / C}\right)^{1 / 2} \breve{S}
$$

where $l_{D}=2^{D} \pi^{D / 2} \Gamma\left(\frac{D}{2}\right)$ is the factor suppressing one-loop diagrams in $D$ dimensions, and $C$ is a group theory factor depending on the unified theory valid above $M_{c}$. The volume 
factor $V_{D-4}$ ensures canonical kinetic terms in $4 \mathrm{D}$ for the zero modes of the bulk fields. In this way, we obtain for the part of the Lagrangian coupling $S$ to the Higgs fields

$$
\begin{aligned}
\mathcal{L}_{D=4} \supset \frac{M^{2}}{l_{4} / C}\left\{\frac{\sqrt{C l_{4}}}{l_{D}}\left[\breve{S}\left(a \breve{H}_{u}^{\dagger} \breve{H}_{d}^{\dagger}+b_{u} \breve{H}_{u}^{\dagger} \breve{H}_{u}+b_{d} \breve{H}_{d}^{\dagger} \breve{H}_{d}\right)+\text { h.c. }\right]_{D}\right. \\
\left.+\frac{C}{l_{D}}\left[\breve{S}^{\dagger} \breve{S}\left(c_{u} \breve{H}_{u}^{\dagger} \breve{H}_{u}+c_{d} \breve{H}_{d}^{\dagger} \breve{H}_{d}+\left(d \breve{H}_{u} \breve{H}_{d}+\text { h.c. }\right)\right)\right]_{D}\right\} .
\end{aligned}
$$

According to NDA, the theory is weakly coupled below the cutoff scale $M$, if all couplings inside the curly brackets in equation (2.12) are smaller than one. This implies the constraints

$$
\begin{gathered}
\frac{\sqrt{C l_{4}}}{l_{D}}\left\{|a|,\left|b_{u}\right|,\left|b_{d}\right|\right\}<1, \\
\frac{C}{l_{D}}\left\{\left|c_{u}\right|,\left|c_{d}\right|,|d|\right\}<1 .
\end{gathered}
$$

Combined with equation (2.10), they translate into the upper bound

$$
\left|A_{0}\right|<\frac{\left\langle F_{S}\right\rangle}{M}\left(\frac{M_{c}}{M}\right)^{D-4} \frac{l_{D}}{\sqrt{C l_{4}}}
$$

on the trilinears. For comparison, the NDA constraint on the gaugino mass is $[6]$

$$
m_{1 / 2}<\frac{\left\langle F_{S}\right\rangle}{M} \frac{1}{2}\left(\frac{M_{c}}{M}\right)^{D-4} \frac{l_{D}}{\sqrt{C l_{4}}} .
$$

Consequently, the ratio of the upper bounds is simply

$$
\frac{\left|A_{0}\right|^{\max }}{m_{1 / 2}^{\max }}=2 .
$$

If the limit on $m_{1 / 2}$ is saturated, it is thus possible for the trilinear couplings to be somewhat larger than the gaugino mass, but not by orders of magnitude.

\section{Phenomenology of the model}

Let us now explore the parameter space of gaugino mediation extended by trilinear couplings. As explained in section 2, the model contains the five free parameters $m_{1 / 2}, m_{H_{u}}^{2}$, $m_{H_{d}}^{2}, A_{0}$, and $B \mu$. The soft squark and slepton masses are negligibly small. This is a realization of the NUHM2 scenario [13] with the restriction $m_{0}=0$. These input parameters are boundary conditions at the compactification scale, which we identify with the scale of gauge coupling unification, $M_{c} \simeq 10^{16} \mathrm{GeV}$. As usual, we trade $B \mu$ for $\tan \beta$ and use the measured $Z$ mass to determine the absolute value of $\mu$. We choose $\mu$ to be positive and restrict ourselves to negative values for $A_{0}$; changing the sign of both parameters would lead to a similar phenomenology.

One of the most important model restrictions is the Higgs mass required to match the value measured at the LHC, see section 3.1 for details. The allowed parameter space accommodates various choices of the lightest sparticle of the MSSM, discussed in section 3.2. 
It comprises the lightest neutralino, the tau sneutrino and the lighter stau. As the latter two are not phenomenologically viable dark matter candidates we assume here that the LSP is a non-MSSM sparticle with very weak interactions. ${ }^{4}$ In the framework of supergravity, this could be the gravitino. In this case the lightest sparticle of the MSSM is the NLSP. Gaugino mediation allows for gravitino masses $m_{3 / 2} \gtrsim 10 \mathrm{GeV}$ [6], in which case the NLSP becomes stable on collider time-scales and the collider signature of the considered model vitally depends on the choice of NLSP. While a neutralino or sneutrino NLSP provides a signature containing missing transverse momentum, detector-stable staus provide a distinct signature of heavy stable charged particles (HSCPs), for which the LHC sensitivity is very high. LHC constraints for the respective signatures are discussed in section 3.3. Bounds from color or charge breaking minima of the scalar potential are briefly discussed in section 3.4. In section 3.5 we comment on the cosmological constraints on the model.

\subsection{Higgs mass}

One of the most important constraints on the parameter space is the experimentally observed Higgs mass of $125.09 \pm 0.24 \mathrm{GeV}$ [3]. The theoretical uncertainty of the Higgs mass prediction in the MSSM is on the order of $\sim 2 \mathrm{GeV}[15,16]$. As the theoretical error is large compared to the experimental one, we do not consider the latter. Furthermore, we assume that the lightest CP-even Higgs of the MSSM plays the role of the observed Higgs. Hence, we consider points with a theoretically predicted mass of the lightest CP-even Higgs in the rage $123 \mathrm{GeV} \lesssim m_{h} \lesssim 127 \mathrm{GeV}$ to be consistent with observations.

In order to compute the Higgs mass we proceed as follows. First we use SPheno 3.3.8 [17, 18] for the calculation of the sparticle masses and low-energy Lagrangian parameters. The output from SPHENo is then used as input to FEYNHigGS 2.12.2 [15, 16, 19-23], which we use to more accurately calculate the lightest Higgs pole mass. Both programs incorporate two-loop diagrams in the calculation of $m_{h}$. However, FeynHiggs 2.12.2 includes a more complete treatment of the calculation, including momentum dependent two-loop QCD contributions [16], leading three-loop contributions [15] and additionally, by combining an effective field theory approach with the fixed-order calculation, it incorporates up to NNLL contributions resummed to all orders [23]. This treatment can significantly reduce the theoretical uncertainties with respect to the pure fixed-order calculation, in particular for large $M_{\text {susy }} \equiv \sqrt{m_{\tilde{t}_{1}} m_{\tilde{t}_{2}}}[23,24]$.

The result for the Higgs mass ${ }^{5}$ is shown in figure 1, where the left panel shows the contour for which $m_{h}=125.09 \mathrm{GeV}$ in the $A_{0}-m_{1 / 2}$ plane. The darker and lighter shaded

\footnotetext{
${ }^{4}$ For the case that a neutralino is the lightest sparticle of the MSSM it could itself be the LSP and hence identified with the dark matter particle. In this case constraints from direct and indirect detection as well as from the thermal relic density could be applied in order to narrow down the viable part of the parameter space. See e.g. [14] for a global fit within the (general) NUHM2 scenario taking into account dark matter observables for a neutralino LSP.

${ }^{5}$ We used the most recent results available in [25] for the Standard Model input parameters relevant for the scans. The values used in both SPheno and FeynHigGs are
}

$$
\begin{aligned}
G_{F} & =1.166379 \cdot 10^{-5} \mathrm{GeV} & m_{b}\left(m_{b}\right) & =4.18 \mathrm{GeV}(\mathrm{SM} \overline{\mathrm{MS}}) \\
m_{Z} & =91.18760 \mathrm{GeV} & m_{\tau} & =1.77686 \mathrm{GeV} \\
\alpha_{s}\left(M_{z}\right) & =1.181 \cdot 10^{-1}(\mathrm{SM} \overline{\mathrm{MS}}) & m_{t} & =1.732 \cdot 10^{2} \mathrm{GeV} \text { (pole mass). }
\end{aligned}
$$



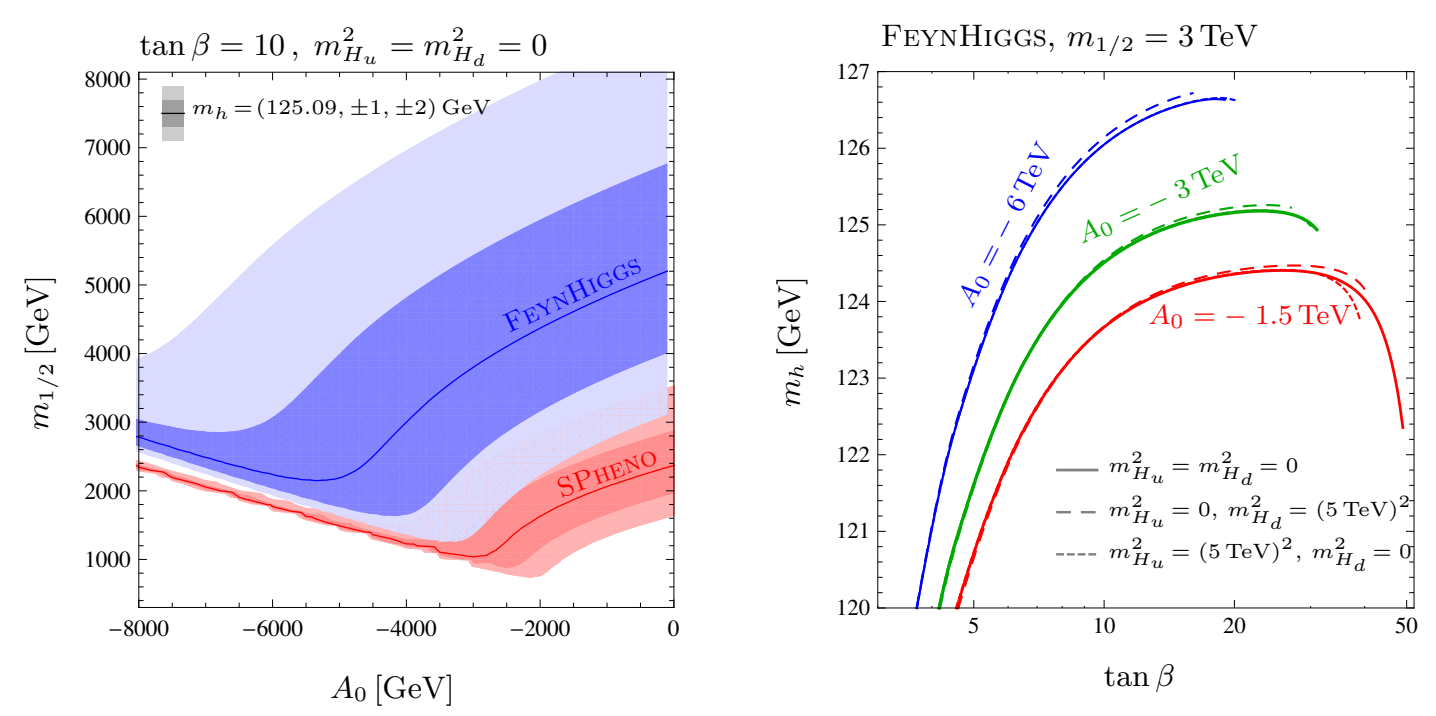

Figure 1. Left panel: contours of the Higgs mass computed by SPHENo (red curve) and FeynHiggs (blue curve) in the $A_{0}-m_{1 / 2}$ plane. The solid lines denote the contour where $m_{h}=125.09 \mathrm{GeV}$ whereas the corresponding darker and lighter shaded areas around them denote a deviation of \pm 1 and $\pm 2 \mathrm{GeV}$, respectively. Right panel: dependence of the Higgs mass, $m_{h}$, computed by FeynHiggs, on $\tan \beta$ for $m_{1 / 2}=3 \mathrm{TeV}$ and three choices of the trilinear coupling $A_{0}=-1.5 \mathrm{TeV}$ (red curves), $A_{0}=-3 \mathrm{TeV}$ (green curves), $A_{0}=-6 \mathrm{TeV}$ (red curves) as well as for three choices of the Higgs soft mass parameters $m_{H_{u}}^{2}=m_{H_{d}}^{2}=0$ (solid curves), $m_{H_{u}}^{2}=0, m_{H_{d}}^{2}=(5 \mathrm{TeV})^{2}$ (long-dashed curves), $m_{H_{u}}^{2}=(5 \mathrm{TeV})^{2}, m_{H_{d}}^{2}=0$ (short-dashed curves).

regions around it denote the \pm 1 and $\pm 2 \mathrm{GeV}$ bands respectively. As mentioned above, we use the Higgs mass as computed by FeynHiggs, represented by the blue curve and bands on the plot. The right panel shows the Higgs mass dependence on $\tan \beta, m_{H_{u}}^{2}$ and $m_{H_{d}}^{2}$ for a fixed value of $m_{1 / 2}$ and three choices of $A_{0}$.

For $\tan \beta=10$ and vanishing $A_{0}$, very large values of $m_{1 / 2}$ on the order of $6 \mathrm{TeV}$ are needed to achieve a suitable Higgs mass of $125 \mathrm{GeV}$. With growing negative $A_{0}$, the required $m_{1 / 2}$ drops to a minimum around $m_{1 / 2} \simeq 2 \mathrm{TeV}$, beyond which the Higgs mass rises again. This minimum corresponds to the maximal mixing scenario, where $\left|X_{t}\right|=$ $\left|A_{t}-\mu \cot \beta\right| \sim \sqrt{6} M_{\text {susy }}$, see [26] for a detailed discussion. This result shows that only with a non-zero trilinear coupling $A_{0}$, a Higgs mass of around $125 \mathrm{GeV}$ can be obtained with $m_{1 / 2}$ such as to obtain a sufficiently light spectrum to be observable in upcoming collider experiments. See further discussion in section 3.3.

The \pm 1 and $\pm 2 \mathrm{GeV}$ bands span a large range, reflecting the relatively large uncertainty in the required value of $m_{1 / 2}$ between 3 and $8 \mathrm{TeV}$. However, this uncertainty band shrinks significantly for large negative $A_{0}$.

The dependence on $\tan \beta$ is shown in the right panel of figure 1. Both very small and very large values of $\tan \beta$ cause the Higgs mass to drop drastically, making it hard to achieve the correct Higgs mass even for very large $m_{1 / 2}$. Note that for large $\tan \beta$ and large negative $A_{0}$, the spectrum acquires tachyonic states. Therefore, not all curves extend to $\tan \beta=50$. 
The influence of the Higgs soft masses $m_{H_{u}}^{2}$ and $m_{H_{d}}^{2}$ on the Higgs mass is small throughout the explored parameter space. The most significant effect arises for large tan $\beta$, cf. the solid and dashed curves in the right panel of figure 1.

The Higgs mass contour as computed by SPHeno, presented by the red curve and shaded bands in the left panel of figure 1, is included for comparison. ${ }^{6}$ The required Higgs mass is reached with considerably smaller $m_{1 / 2}$ for a given $A_{0}$, as the SPHENO result for $m_{h}$ is typically around $3 \mathrm{GeV}$ larger than the one from FEYNHigGs. In particular for large $M_{\text {susy }}$, NNLL resummation can yield important corrections that significantly contribute to the difference between the results obtained by the two codes, see e.g. [16, 23, 24] for details.

\subsection{Particle spectrum}

The phenomenology of the model regarding collider searches, astrophysics and cosmology strongly depends on the nature of the NLSP. As mentioned above, we compute the sparticle spectrum with SPHEno. In the considered parameter space, we encounter three possible candidates for the NLSP: the neutralino, the sneutrino, or the lighter stau, which can be predominantly left- or right handed. Figure 2 shows several projections of the parameter space in the plane $m_{H_{d}}^{2} / m_{1 / 2}^{2}-A_{0} / m_{1 / 2}$. We have rescaled $m_{H_{d}}$ and $A_{0}$ by $m_{1 / 2}$ as the nature of the NLSP is almost independent of the overall mass scale that is governed mostly by $m_{1 / 2}$. In other words, for fixed ratios $A_{0} / m_{1 / 2}, m_{H_{d}}^{2} / m_{1 / 2}^{2}$ and $m_{H_{u}}^{2} / m_{1 / 2}^{2}$, the sparticle spectrum is mainly shifted with $m_{1 / 2}$ and the shown projections remain approximately unchanged.

The results summarized in figure 2 demonstrate the relationship between the Higgs soft masses and the NLSP. As the ratio $r \equiv\left(m_{H_{u}}^{2}-m_{H_{d}}^{2}\right) / m_{1 / 2}^{2}$ becomes more negative, the NLSP can shift from the stau, to the neutralino and finally to the sneutrino, depending on the value of $\tan \beta$ and $A_{0}$. If $\tan \beta$ is relatively large and $A_{0}$ is large and negative, only a stau NLSP is possible. Interestingly, the stau NLSP is also observed to shift through regions of right-chirality, large mixing and left-chirality with decreasing $r$ (cf. the gray solid curve in the plots of figure 3 , showing the stau mixing angle). In addition, figure 2 depicts the NLSP sensitivity to the value of $\tan \beta$, showing that the stau NLSP region grows with $\tan \beta$. In fact, for $\tan \beta \gtrsim 30$, the entire region contains only a stau NLSP. We also find that some of the regions of interest contain unphysical tachyonic spectra, meaning negative soft-masses squared. This occurs when $A_{0}$ has a large negative value compared to $m_{1 / 2}$, and becomes more frequent with increasing $\tan \beta$.

We would like to explain some of this behavior in a rough analytical manner, beginning with the chirality switch of the stau. This can be understood from analyzing the one-loop RGE's for the third generation leptonic soft masses [11]

$$
\begin{aligned}
16 \pi^{2} \frac{d}{d t} m_{L_{3}}^{2} & =\chi_{\tau}-6 g_{2}^{2}\left|M_{2}\right|^{2}-\frac{6}{5} g_{1}^{2}\left|M_{1}\right|^{2}-\frac{3}{5} g_{1}^{2} \Sigma \\
16 \pi^{2} \frac{d}{d t} m_{\bar{e}_{3}}^{2} & =2 \chi_{\tau}-\frac{24}{5} g_{1}^{2}\left|M_{1}\right|^{2}+\frac{6}{5} g_{1}^{2} \Sigma,
\end{aligned}
$$

\footnotetext{
${ }^{6}$ For definiteness we also show $\pm 2 \mathrm{GeV}$ bands for the SPHENo predicition. However, the actual uncertainty might be larger [24].
} 

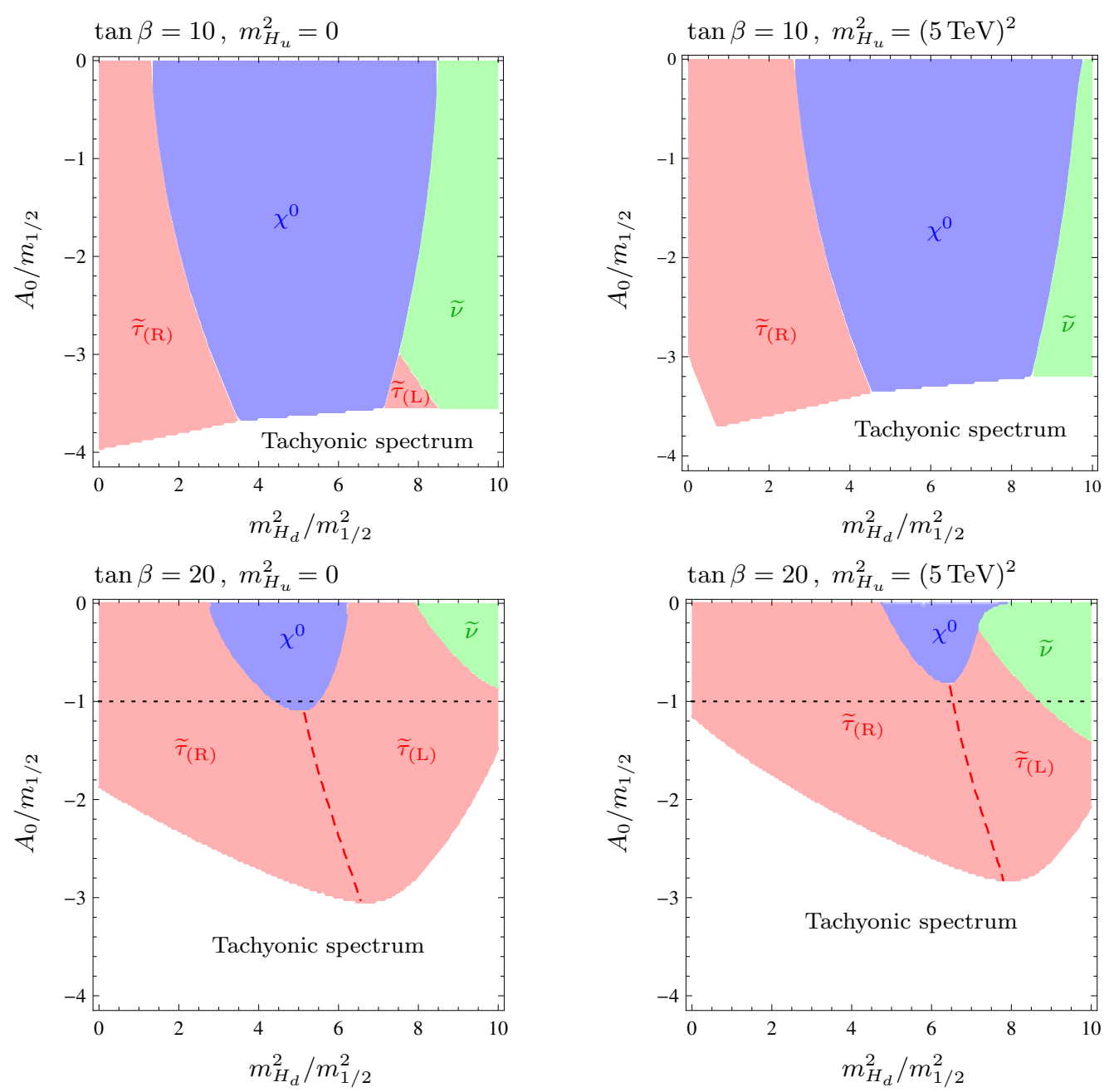

Figure 2. Regions characterized by a stau (red), neutralino (blue) and sneutrino (green) NLSP in the $m_{H_{d}}^{2} / m_{1 / 2}^{2}-A_{0} / m_{1 / 2}$ plane for four choices of $\tan \beta$ and $m_{H_{u}}^{2}$. All panels have $m_{1 / 2}=2 \mathrm{TeV}$. In the white region below, we run into a tachyonic region, i.e., negative soft masses squared. The red dashed curve indicates the transition from a predominantly right- to left-handed stau NLSP, i.e., the contour $\sin ^{2} \theta_{\tau}=1 / 2$. The black dotted lines in the lower plots denote the slices in parameter space that are considered in figure 3 .

where

$$
\begin{aligned}
\chi_{\tau} & \equiv 2\left|y_{\tau}\right|^{2}\left(m_{H_{d}}^{2}+m_{L_{3}}^{2}+m_{\bar{e}_{3}}^{2}\right)+2\left|a_{\tau}\right|^{2} \\
\Sigma & \equiv m_{H_{u}}^{2}-m_{H_{d}}^{2}+\operatorname{Tr}\left[m_{Q}^{2}-m_{L}^{2}-2 m_{\bar{u}}^{2}+m_{\bar{d}}^{2}+m_{\bar{e}}^{2}\right]
\end{aligned}
$$

For $m_{H_{d}}^{2} \gg m_{1 / 2}$, we can neglect the gaugino masses in the above formula, and the running will depend mostly on the $\Sigma$ parameter. From equation (3.2), one sees that for very large $m_{H_{d}}^{2}$, this value is negative, and will therefore lower the value of the left-chiral soft mass term but increase the size of the right-chiral term. Therefore, the NSLP will become more left-chiral with increasing $m_{H_{d}}^{2}$. For larger values of $m_{H_{u}}^{2}$, the absolute value of the $\Sigma$ term is smaller, and the progression from right- to left-chirality happens at larger values of $m_{H_{d}}^{2}$. 

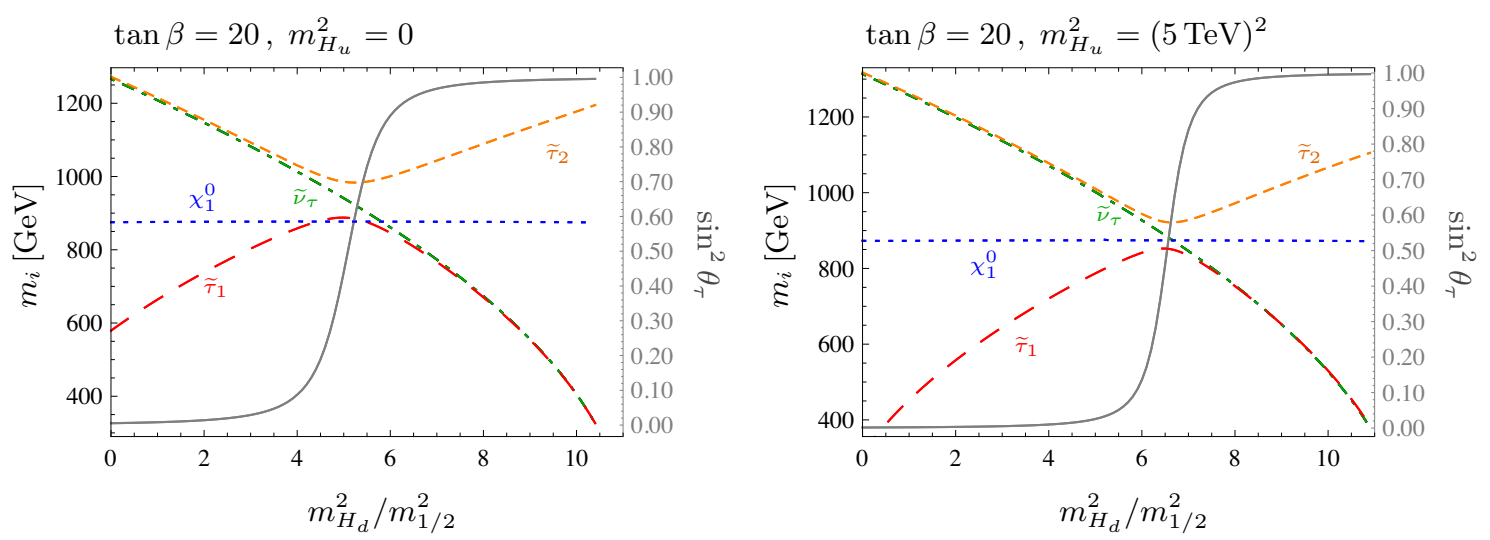

Figure 3. Sparticle masses $m_{\widetilde{\tau}_{1}}$ (red long-dashed curve), $m_{\widetilde{\tau}_{2}}$ (orange short-dashed curve), $m_{\widetilde{\nu}_{\tau}}$ (green dot-dashed curve) and $m_{\chi_{1}^{0}}$ (blue dotted curve) as a function of $m_{H_{d}}^{2} / m_{1 / 2}^{2}$ for two choices of $\tan \beta$ and $m_{H_{u}}^{2}$. The stau mixing angle is indicated through the gray solid line showing $\sin ^{2} \theta_{\tau}$, labelled on the right axis.

Regions where the sneutrino becomes the LSP are also determined by equations (3.1). Again, these regions occur in the limit $m_{H_{d}}^{2} \gg m_{1 / 2}$, so we can make the same approximation and assume that the stau is mostly left-chiral. When the stau is mostly left-chiral, it is a delicate matter which of the two particles becomes the NLSP. The sneutrino mass is completely determined by equation (3.1a), as there are no right-chiral neutrinos in the MSSM, whereas there is mixing in the stau sector. The off-diagonal elements in the stau mixing matrix, which are $A_{0}$ and $\tan \beta$ dependent, push the eigenvalue down. However, the diagonal elements, which are predominantly dependent on the soft masses $m_{L_{3}}^{2}$ and $m_{\bar{e}_{3}}^{2}$, but also depend on the "hyperfine splitting" arising from EWSB, increase the eigenvalues. In figure 3 we show the masses of the staus, the tau sneutrino and the neutralino for the two slices denoted by the black dotted lines in the lower panels of figure 2 . It reveals the small mass difference between $\widetilde{\tau}_{1}$ and $\widetilde{\nu}_{\tau}$ for large $m_{H_{d}}^{2} / m_{1 / 2}^{2}$.

The $\tan \beta$ and $A_{0}$ dependence can be understood by first noting that the neutralino mass is pushed up with $\tan \beta$, and larger values of $A_{0}$ push the third generation leptonic soft masses down by increasing $\chi_{\tau}$. This explains the shrinking neutralino region seen in the lower panels of figure 2. Large values of $A_{0}$ also increase mixing in the stau sector, pushing down the smallest eigenvalue of the stau mass matrix, implying the sneutrino LSP region should also shrink with larger $\tan \beta$.

\subsection{Tests at colliders}

Heavy stable charged particles. The lighter stau is the NLSP for a large part of the considered parameter space in our model. In order to determine the 95\% CL exclusion limits from collider searches for HSCPs, we first compute the total cross section for the production of sparticles with Pyтнia 6 [27]. For points with $\sigma_{8 \mathrm{TEV}}^{\text {tot }}>1 / \mathcal{L}_{8 \mathrm{TEV}}^{\text {int }}$, i.e. for an expected total signal of more than one event we perform a Monte Carlo simulation of the signal at the $8 \mathrm{TeV}$ LHC with the MADGRAPH5_AMC@NLO event generator [28]. We generate $10 \mathrm{k}$ events for each point in the model parameter space, taking into account 
all possible sparticle production channels. The decay, showering and hadronization is performed with Pyтhia 6 [27]. We do not perform a detector simulation. Instead we determine the signal efficiencies with the method introduced in ref. [29], which allows for the direct analysis of the hadron-level events on the basis of the kinematic properties of isolated HSCP candidates. In order to identify isolated HSCP candidates we first impose the isolation criteria

$$
\left(\sum_{i}^{\substack{\text { charged particles } \\ \Delta R<0.3}} p_{\mathrm{T}}^{i}\right)<50 \mathrm{GeV}
$$

and

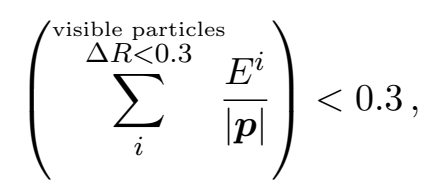

where the sums include all charged and visible particles, respectively, in a cone of $\Delta R=\sqrt{\Delta \eta^{2}+\Delta \phi^{2}}<0.3$ around the direction of the HSCP candidate, $p_{\mathrm{T}}{ }^{i}$ denotes their transverse momenta and $E^{i}$ their energy. Muons are not considered as visible particles as their energy deposition in the calorimeter is small. $|\boldsymbol{p}|$ is the magnitude of the three-momentum of the HSCP candidate. The HSCP candidate itself is not included in either sum.

We compute the signal efficiency by averaging the probabilities for events to pass the on- and off-line selection criteria [29],

$$
\epsilon=\frac{1}{N} \sum_{i}^{N} P_{\mathrm{on}, i}^{(n)} \times P_{\mathrm{off}, i}^{(n)},
$$

where the sum runs over all $N$ generated events $i$. For events containing one or two HSCP candidates the probabilities are given by

$$
P_{\text {on } / \mathrm{off}, i}^{(1)}=P_{\text {on } / \mathrm{off}}\left(\boldsymbol{k}_{i}^{1}\right)
$$

or

$$
P_{\text {on/off }, i}^{(2)}=P_{\text {on } / \text { off }}\left(\boldsymbol{k}_{i}^{1}\right)+P_{\text {on/off }}\left(\boldsymbol{k}_{i}^{2}\right)-P_{\text {on/off }}\left(\boldsymbol{k}_{i}^{1}\right) P_{\text {on/off }}\left(\boldsymbol{k}_{i}^{2}\right),
$$

respectively, where $\boldsymbol{k}_{i}^{1,2}$ are the kinematical vectors of the HSCP candidates in the $i$ th event. $\boldsymbol{k}=\left(\eta, p_{\mathrm{T}}, \beta\right)$ contains the candidate's pseudo-rapidity, $\eta$, transverse momentum, $p_{\mathrm{T}}$, and velocity, $\beta$.

The CMS analysis [29] requires a minimum reconstructed mass, $m_{\mathrm{rec}}$, for the candidate. The probabilities $P_{\text {on/off }}(\boldsymbol{k})$ are provided for four distinct mass cuts

$$
m_{\text {rec }}>0,100,200,300 \mathrm{GeV} \text {, }
$$

which we here consider to be four different signal regions. Due to detector resolution effects, the reconstructed mass is typically $m_{\mathrm{rec}} \simeq 0.6 m_{\mathrm{HSCP}}$ [29]. Hence, we set the efficiencies to zero if $0.6 m_{\mathrm{HSCP}}$ is below the respective mass cut of the signal region. 

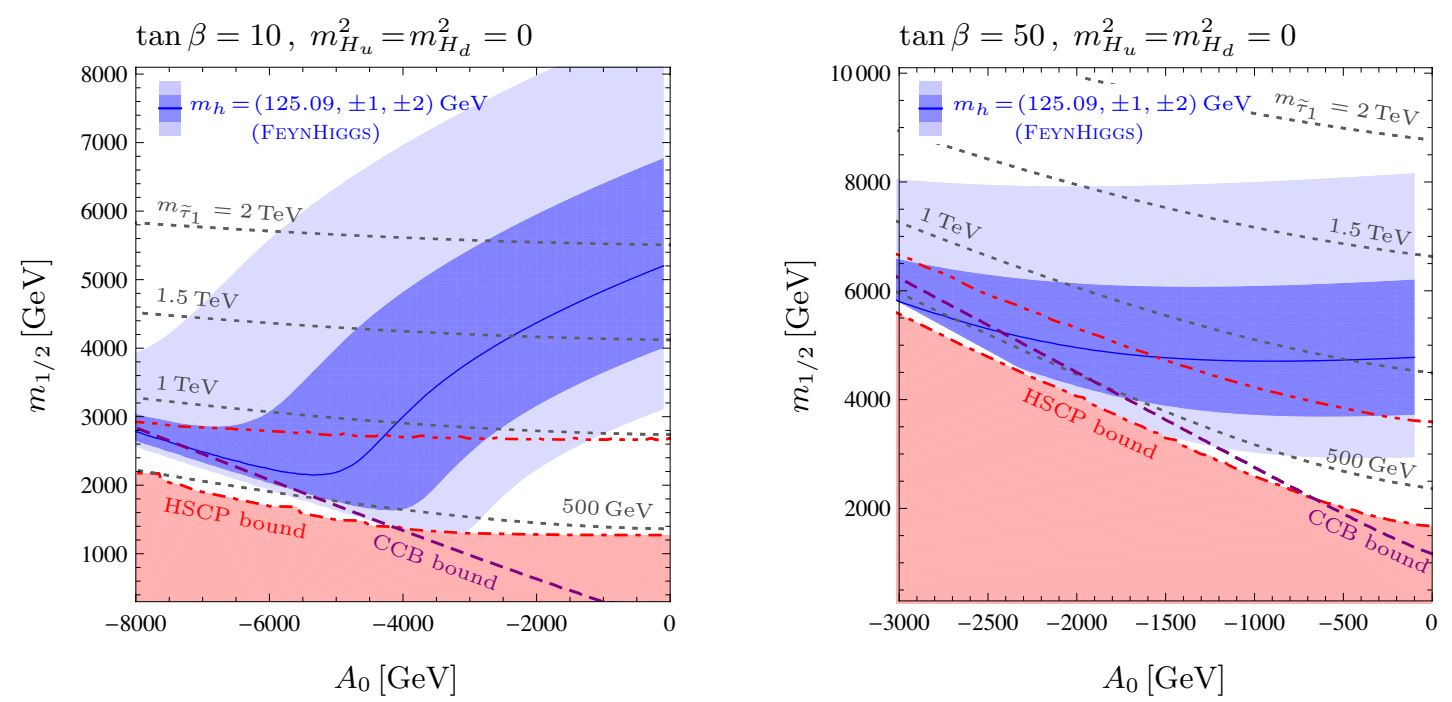

Figure 4. Contours of $m_{h}=125.09 \mathrm{GeV}$ computed by FeynHigGs (blue solid curve) in the $A_{0^{-}}$ $m_{1 / 2}$ plane, as well as constraints from searches for heavy stable charged particles (HSCP) at the $8 \mathrm{TeV}$ LHC (red shaded region below the red dot-dashed curve). Projections for the $13 \mathrm{TeV}$ LHC at $300 \mathrm{fb}^{-1}$ are indicated by the red dot-dot-dashed curve. The purple dashed line represents the strongest of the CCB constraints from equations (3.9)-(3.10). The grey dotted curves show the contours of the lighter stau mass $m_{\widetilde{\tau}_{1}}$. For $\tan \beta=50$ and $-A_{0} \gtrsim 2.3 \mathrm{TeV}$ the HSCP limit (dotdashed curve) extents into the region of a tachyonic spectrum, in this region this limit is only an extrapolation.

This prescription is also used in ref. [30], where it is validated by reproducing the efficiencies and cross section upper limits for the gauge mediated supersymmetry breaking model from the full CMS detector simulation [29] with a relative error below $5 \%$.

The resulting limits are shown in figure 4 , projected onto the $A_{0}-m_{1 / 2}$ plane for two slices in parameter space, where $m_{H_{u}}^{2}=m_{H_{d}}^{2}=0$, and $\tan \beta=10$ (left panel) and $\tan \beta=$ 50 (right panel). Both choices are characterized by a stau NLSP in the entire considered parameter plane. The considered CMS search for HSCPs at the $8 \mathrm{TeV}$ LHC excludes the region below the red dot-dashed line (red shaded region) at 95\% CL. The exclusion reach depends strongly on the overall sparticle mass spectrum, which is indicated by drawing several contours for the mass of the stau NLSP. The exclusion limits turn out to cut at around $m_{\widetilde{\tau}_{1}} \gtrsim 400 \mathrm{GeV}$ with a mild dependence on the other parameters. This translates into a limit on $m_{1 / 2}$ between 1 and $2 \mathrm{TeV}$ for $\tan \beta=10$ in the considered region of $A_{0}$, but can be much larger for large $\tan \beta$, as shown in the right panel. The existing limit only touches the $-2 \mathrm{GeV}$ band regarding the Higgs mass, and leaves most of the parameter space that provides a Higgs mass in the range $123 \mathrm{GeV} \lesssim m_{h} \lesssim 127 \mathrm{GeV}$ unchallenged.

The $13 \mathrm{TeV}$ LHC runs have pursued searches for heavy stable charged particles, and (preliminary) results from an integrated luminosity of $2.5 \mathrm{fb}^{-1}$ [31] (12.9 $\mathrm{fb}^{-1}$ [32]) have been released. For the $13 \mathrm{TeV}$ searches, no on-/off-line probabilities (as in ref. [29]), have been provided, such that these searches cannot be easily reinterpreted. We do, however, expect to obtain a meaningful estimate of the $13 \mathrm{TeV}$ sensitivity as described in the following. 
The signal efficiencies for the $8 \mathrm{TeV}$ LHC increase with increasing $m_{\widetilde{\tau}_{1}}$ for the tested points, and are only mildly dependent on the other parameters within the considered model. In particular, we found that the efficiency is always above 0.5 for $m_{\widetilde{\tau}_{1}}>350 \mathrm{GeV}$, and above 0.6 for $m_{\widetilde{\tau}_{1}}>500 \mathrm{GeV}$ in our scan. Assuming a similar detector performance, the efficiencies at the $13 \mathrm{TeV}$ LHC for a certain stau mass will to first approximation be the same as for the $8 \mathrm{TeV}$ efficiency, for a mass that is smaller by a factor of $8 / 13$. Hence, for the $13 \mathrm{TeV}$ LHC we assume an efficiency of 0.5 , which is expected to provide a mostly conservative estimate for stau masses above $600 \mathrm{GeV}$. Furthermore, as for $m_{\text {rec }}>200 \mathrm{GeV}$ the signal region is typically background-free $[29,31]$ we require 3 signal events in the signal region supporting a $95 \%$ CL exclusion limit. In this way we estimated the projected sensitivity for $300 \mathrm{fb}^{-1}$ at $13 \mathrm{TeV}$, for which we computed the production cross sections with Pyтнia 6 [27], see the red dot-dot-dashed curves in figure 4. The projected exclusion reach cuts into a larger portion of the parameter space providing the correct Higgs mass. In particular, the maximal mixing scenario for moderate values for $\tan \beta$ can be tested. With $300 \mathrm{fb}^{-1}$, stau masses up to around $1 \mathrm{TeV}$ could be tested.

Note that performing the same estimate for the analysis at $2.5 \mathrm{fb}^{-1}\left(12.9 \mathrm{fb}^{-1}\right)$ provides an estimated limit very close to (slightly above) the $8 \mathrm{TeV}$ limit, which we do not show in figure 4 for the sake of better readability.

Missing energy signatures. As discussed in section 3.2, a high enough $m_{H_{d}}^{2}$ relative to $m_{H_{u}}^{2}$ and $m_{1 / 2}$ results in a neutralino or even sneutrino NLSP. If present in collision events, neutral NLSPs lead to a missing transverse energy (MET) signature at the LHC.

In order to test the compatibility with current LHC results, we perform a Monte Carlo simulation with the MADGRAPH5_AMC@NLO event generator [28] for the $8 \mathrm{TeV}$ LHC. We generate 20k events. The decay, showering and hadronization is performed by Pythia 6 [27]. The results are used as input to CheckMate $1[33],{ }^{7}$ allowing us to simultaneously test the signal against various LHC searches for missing transverse energy.

We test our model against all ATLAS analyses implemented in CHECKMATE 1 [38-58]. These analyses search for final states containing a significant amount of missing transverse energy, in addition to jets or leptons. The signal is compared to experimental limits in the respective signal regions of the analysis at $95 \% \mathrm{CL}$. The most sensitive region from all the analyses is used to conclude whether the model can be excluded or not. Among the points that provide a Higgs mass $m_{h}>123 \mathrm{GeV}$, we tested the lighter part of the spectrum, i.e., $m_{1 / 2} \leq 3 \mathrm{TeV}$ for various slices in parameter space regarding $\tan \beta, A_{0}$ and Higgs soft masses. We found that even for the lightest spectra the signal falls below the exclusion limits by at least an order of magnitude. Since the spectrum becomes heavier for larger values of $m_{1 / 2}$, we expect no sensitivity of searches for MET in the region $m_{h}>123 \mathrm{GeV}$. The analysis which most frequently has the largest signal region is the search for direct stop pair production in final states with two leptons [38].

\footnotetext{
${ }^{7}$ CheckMate is built upon a number of external tools. The detector simulation is based on Delphes 3 [34], which incorporates FASTJET [35, 36] using the Anti-kt jet algorithm [37].
} 


\subsection{Charge and color breaking}

In addition to the collider constraints, we investigate whether and in which regions of parameter space the current model is limited by charge- and color-breaking minima of the scalar potential. The MSSM contains 26 scalars, most of which carry electric or color charge. Hence, there is a danger of introducing charge- and color-breaking (CCB), depending on their VEVs where the scalar potential has its minimum. Due to the large number of scalars in the theory, the scalar potential is very complex, limiting an analytical approach to only considering certain rays in field space. It is common to investigate directions in field space where the VEVs of the Higgses and $\tilde{\tau}_{L / R}$ or $\tilde{t}_{L / R}$ have the same value, and to neglect the $D$-term of the potential, which is a gauge interaction and positive for non-zero values of the scalar fields, as well as loop corrections. Based on criteria for CCB as found in [59-61], we use the same condition as [5] for the stop trilinear coupling, namely

$$
A_{t}^{2}<3\left(m_{H_{u}}^{2}+|\mu|^{2}+m_{Q_{3}}^{2}+m_{\bar{u}_{3}}^{2}\right) .
$$

By analogy, we take the bound on the stau trilinear to be

$$
A_{\tau}^{2}<3\left(m_{H_{d}}^{2}+|\mu|^{2}+m_{L_{3}}^{2}+m_{\bar{e}_{3}}^{2}\right) .
$$

For large $\tan \beta$, one can derive an upper bound on the product $\mu \tan \beta$ requiring the standard electroweak vacuum to be stable or metastable with a lifetime larger than the age of the universe [62-65]. We use [65],

$$
\begin{aligned}
\left|\mu \tan \beta_{\mathrm{eff}}\right|< & 56.9 \sqrt{m_{L_{3}} m_{\bar{e}_{3}}}+57.1\left(m_{L_{3}}+1.03 m_{\bar{e}_{3}}\right)-1.28 \times 10^{4} \mathrm{GeV} \\
+ & \frac{1.67 \times 10^{6} \mathrm{GeV}^{2}}{m_{L_{3}}+m_{\bar{e}_{3}}}-6.41 \times 10^{7} \mathrm{GeV}^{3}\left(\frac{1}{m_{L_{3}}^{2}}+\frac{0.983}{m_{\bar{e}_{3}}^{2}}\right),
\end{aligned}
$$

where $\tan \beta_{\text {eff }} \equiv \tan \beta /\left(1+\Delta_{\tau}\right)$ with

$$
\Delta_{\tau} \simeq-\frac{3 g^{2}}{32 \pi^{2}} \mu \tan \beta M_{2} I\left(m_{\widetilde{\nu}_{\tau}}, M_{2}, \mu\right)+\frac{g^{\prime 2}}{16 \pi^{2}} \mu \tan \beta M_{1} I\left(m_{\widetilde{\tau}_{1}}, m_{\widetilde{\tau}_{2}}, M_{1}\right),
$$

and

$$
I(a, b, c)=\frac{1}{\left(a^{2}-b^{2}\right)\left(b^{2}-c^{2}\right)\left(a^{2}-c^{2}\right)}\left(a^{2} b^{2} \log \frac{a^{2}}{b^{2}}+b^{2} c^{2} \log \frac{b^{2}}{c^{2}}+c^{2} a^{2} \log \frac{c^{2}}{a^{2}}\right) .
$$

These bounds are superimposed in figure 4, where we show the most constraining bound from equations (3.9)-(3.10). For $\tan \beta=10$, the region below the purple dashed line violates equation (3.9), while for $\tan \beta=50$ it violates equation (3.10). For large negative $A_{0}$, the CCB bound cuts into the part of the parameter space that provides the correct Higgs mass.

Note that we impose these bounds as a first estimate, indicating the region where CCB constraints might exclude points in the parameter space. It has been shown $[66,67]$ that these bounds are useful, but not entirely reliable in determining vacuum stability when more sophisticated analyses are performed. We leave a detailed numerical analysis of the vacuum stability utilizing Vevacious [68] for future work. 


\subsection{Cosmological constraints}

Scenarios with long-lived NLSPs are subject to constraints from big bang nucleosynthesis (BBN) because the presence and late decays of the NLSPs can change the primordial abundances of light elements [69-71]. In our case, the NLSP decays comparatively early on BBN timescales due to the relatively heavy sparticle spectrum. For example, for gaugino mediation with a stau NLSP a lower bound of $m_{\widetilde{\tau}} \gtrsim 400 \mathrm{GeV}$ was found in [72], which roughly coincides with the lower limit from HSCP searches. Therefore we do not perform a detailed analysis here.

Another constraint we did not include is the non-thermal production of gravitino dark matter by NLSP decays, which may not exceed the observed dark matter density. This is interesting from a theoretical point of view because it leads to an upper bound on the sparticle masses but less relevant for phenomenology, since the constraint becomes relevant only for very large values of $m_{1 / 2}$ [72], which are far beyond the reach of the LHC.

\section{Conclusions}

We have considered phenomenological constraints on the gaugino mediation model of supersymmetry breaking. First, we verified that the model allows for soft trilinear scalar interaction terms. These terms were originally assumed to vanish in gaugino mediation and play a crucial role in achieving a Higgs mass in agreement with the observed value of $125 \mathrm{GeV}$. The trilinear matrices are proportional to the Yukawa coupling matrices, thus avoiding flavor problems. The proportionality factor can be different for up- and downtype sfermions.

Second, we explored the phenomenological consequences of non-vanishing trilinears. The first constraint we discussed is the experimentally observed Higgs mass, calculating the low-energy parameters and the sparticle spectrum with SPHENO and the Higgs mass with FeynHiggs. We determined the parameter space regions where the Higgs mass lies within the LHC limits. Large negative trilinears are required to obtain an acceptable Higgs mass if the SUSY scale is to be kept near the reach of the LHC. We also observe that FEYNHIGGS 2.12.2 - incorporating important NNLL contributions - predict a Higgs mass around $3 \mathrm{GeV}$ lower compared to the SPHENO calculation in the parameter regions considered.

We also considered the phenomenological implications of the non-universal soft Higgs masses. We found that these parameters mainly affect which sparticle becomes the NLSP (we assume a gravitino LSP and that the lightest MSSM sparticle is the NLSP). Values of the ratio $r \equiv\left(m_{H_{u}}^{2}-m_{H_{d}}^{2}\right) / m_{1 / 2}^{2}$ near zero correspond to a stau NLSP. As $r$ is pushed to larger negative values, the NLSP can become the neutralino and eventually the tau sneutrino. This behavior also depends on $A_{0}$ and $\tan \beta$. For sufficiently large $\left|A_{0}\right|$ and $\tan \beta$, the composition of the stau NLSP changes from mainly $\widetilde{\tau}_{\mathrm{R}}$ to mainly $\widetilde{\tau}_{\mathrm{L}}$ as $r$ becomes large and negative, passing through regions with large mixing.

Proceeding to investigate the LHC sensitivity of the scenario, we found that for a neutral NLSP, the viable part of parameter space is not challenged by missing energy searches. However, for a stau NLSP, the corresponding searches for heavy stable charged 
particles become sensitive and cut into the region where $123 \mathrm{GeV} \lesssim m_{h} \lesssim 127 \mathrm{GeV}$. The projection for an integrated luminosity of $300 \mathrm{fb}^{-1}$ reaches a large portion of this part of parameter space, especially in the maximal-mixing scenario.

Finally, we indicate in which regions of parameter space the model might be limited by charge- and color-breaking minima of the scalar potential by using (semi-)analytic estimates for the CCB conditions. It turns out that only a small part of the allowed Higgs mass region is in conflict with these CCB bounds.

\section{Acknowledgments}

We would like to thank Sven Heinemeyer, Ben O'Leary, Werner Porod, Florian Staub, Jamie Tattersall, and Alexander Voigt for helpful discussions. Special thanks are due to Felix Brümmer for pointing out to us the possibility of non-zero trilinear couplings in gaugino mediation. We acknowledge support by the German Research Foundation (DFG) through the research unit "New physics at the LHC".

Open Access. This article is distributed under the terms of the Creative Commons Attribution License (CC-BY 4.0), which permits any use, distribution and reproduction in any medium, provided the original author(s) and source are credited.

\section{References}

[1] D.E. Kaplan, G.D. Kribs and M. Schmaltz, Supersymmetry breaking through transparent extra dimensions, Phys. Rev. D 62 (2000) 035010 [hep-ph/9911293] [INSPIRE].

[2] Z. Chacko, M.A. Luty, A.E. Nelson and E. Ponton, Gaugino mediated supersymmetry breaking, JHEP 01 (2000) 003 [hep-ph/9911323] [INSPIRE].

[3] ATLAS and CMS collaborations, Combined measurement of the Higgs boson mass in pp collisions at $\sqrt{s}=7$ and 8 TeV with the ATLAS and CMS experiments, Phys. Rev. Lett. 114 (2015) 191803 [arXiv: 1503.07589] [INSPIRE].

[4] R. Kitano, R. Motono and M. Nagai, MSSM without free parameters, Phys. Rev. D 94 (2016) 115016 [arXiv: 1605.08227] [INSPIRE].

[5] F. Brummer, S. Kraml and S. Kulkarni, Anatomy of maximal stop mixing in the MSSM, JHEP 08 (2012) 089 [arXiv: 1204.5977] [INSPIRE].

[6] W. Buchmüller, K. Hamaguchi and J. Kersten, The gravitino in gaugino mediation, Phys. Lett. B 632 (2006) 366 [hep-ph/0506105] [INSPIRE].

[7] H. Pagels and J.R. Primack, Supersymmetry, cosmology and new TeV physics, Phys. Rev. Lett. 48 (1982) 223 [INSPIRE].

[8] W. Buchmüller, J. Kersten and K. Schmidt-Hoberg, Squarks and sleptons between branes and bulk, JHEP 02 (2006) 069 [hep-ph/0512152] [INSPIRE].

[9] Z. Chacko, M.A. Luty and E. Ponton, Massive higher dimensional gauge fields as messengers of supersymmetry breaking, JHEP 07 (2000) 036 [hep-ph/9909248] [INSPIRE].

[10] A. Brignole, L.E. Ibáñez and C. Muñoz, Soft supersymmetry breaking terms from supergravity and superstring models, Adv. Ser. Direct. High Energy Phys. 18 (1998) 125 [hep-ph/9707209] [INSPIRE]. 
[11] S.P. Martin, A Supersymmetry primer, World Scientific, Singapore (2011), hep-ph/9709356 [INSPIRE].

[12] D. Matalliotakis and H.P. Nilles, Implications of nonuniversality of soft terms in supersymmetric grand unified theories, Nucl. Phys. B 435 (1995) 115 [hep-ph/9407251] [INSPIRE].

[13] J.R. Ellis, K.A. Olive and Y. Santoso, The MSSM parameter space with nonuniversal Higgs masses, Phys. Lett. B 539 (2002) 107 [hep-ph/0204192] [INSPIRE].

[14] O. Buchmueller et al., The NUHM2 after LHC Run 1, Eur. Phys. J. C 74 (2014) 3212 [arXiv: 1408.4060] [INSPIRE].

[15] T. Hahn, S. Heinemeyer, W. Hollik, H. Rzehak and G. Weiglein, High-precision predictions for the light CP-even Higgs boson mass of the minimal supersymmetric standard model, Phys. Rev. Lett. 112 (2014) 141801 [arXiv:1312.4937] [INSPIRE].

[16] S. Borowka, T. Hahn, S. Heinemeyer, G. Heinrich and W. Hollik, Momentum-dependent two-loop QCD corrections to the neutral Higgs-boson masses in the MSSM, Eur. Phys. J. C 74 (2014) 2994 [arXiv: 1404.7074] [INSPIRE].

[17] W. Porod, SPheno, a program for calculating supersymmetric spectra, SUSY particle decays and SUSY particle production at $e^{+} e^{-}$colliders, Comput. Phys. Commun. 153 (2003) 275 [hep-ph/0301101] [INSPIRE].

[18] W. Porod and F. Staub, SPheno 3.1: Extensions including flavour, CP-phases and models beyond the MSSM, Comput. Phys. Commun. 183 (2012) 2458 [arXiv:1104.1573].

[19] M. Frank, T. Hahn, S. Heinemeyer, W. Hollik, H. Rzehak and G. Weiglein, The Higgs boson masses and mixings of the complex MSSM in the Feynman-diagrammatic approach, JHEP 02 (2007) 047 [hep-ph/0611326] [INSPIRE].

[20] G. Degrassi, S. Heinemeyer, W. Hollik, P. Slavich and G. Weiglein, Towards high precision predictions for the MSSM Higgs sector, Eur. Phys. J. C 28 (2003) 133 [hep-ph/0212020] [INSPIRE].

[21] S. Heinemeyer, W. Hollik and G. Weiglein, The Masses of the neutral CP-even Higgs bosons in the MSSM: accurate analysis at the two loop level, Eur. Phys. J. C 9 (1999) 343 [hep-ph/9812472] [INSPIRE].

[22] S. Heinemeyer, W. Hollik and G. Weiglein, FeynHiggs: a program for the calculation of the masses of the neutral CP even Higgs bosons in the MSSM, Comput. Phys. Commun. 124 (2000) 76 [hep-ph/9812320].

[23] H. Bahl and W. Hollik, Precise prediction for the light MSSM Higgs boson mass combining effective field theory and fixed-order calculations, Eur. Phys. J. C 76 (2016) 499 [arXiv: 1608.01880] [INSPIRE].

[24] P. Athron, J.-h. Park, T. Steudtner, D. Stöckinger and A. Voigt, Precise Higgs mass calculations in (non-)minimal supersymmetry at both high and low scales, JHEP 01 (2017) 079 [arXiv: 1609.00371] [INSPIRE].

[25] Particle Data Group collaboration, K.A. Olive et al., Review of particle physics, Chin. Phys. C 38 (2014) 090001 [inSPIRE].

[26] M. Carena et al., Reconciling the two loop diagrammatic and effective field theory computations of the mass of the lightest CP-even Higgs boson in the MSSM, Nucl. Phys. B 580 (2000) 29 [hep-ph/0001002] [INSPIRE].

[27] T. Sjöstrand, S. Mrenna and P.Z. Skands, PYTHIA 6.4 physics and manual, JHEP 05 (2006) 026 [hep-ph/0603175] [inSPIRE]. 
[28] J. Alwall et al., The automated computation of tree-level and next-to-leading order differential cross sections and their matching to parton shower simulations, JHEP 07 (2014) 079 [arXiv: 1405.0301] [INSPIRE].

[29] CMS collaboration, Constraints on the pMSSM, AMSB model and on other models from the search for long-lived charged particles in proton-proton collisions at $\sqrt{s}=8 \mathrm{TeV}$, Eur. Phys. J. C 75 (2015) 325 [arXiv: 1502.02522] [INSPIRE].

[30] J. Heisig, A. Lessa and L. Quertenmont, Simplified models for exotic BSM searches, JHEP 12 (2015) 087 [arXiv: 1509.00473] [INSPIRE].

[31] CMS collaboration, Search for long-lived charged particles in proton-proton collisions at $\sqrt{s}=13 \mathrm{TeV}$, Phys. Rev. D 94 (2016) 112004 [arXiv:1609.08382] [InSPIRE].

[32] CMS collaboration, Search for heavy stable charged particles with $12.9 \mathrm{fb}^{-1}$ of 2016 data, CMS-PAS-EXO-16-036 (2016).

[33] M. Drees, H. Dreiner, D. Schmeier, J. Tattersall and J.S. Kim, CheckMATE: confronting your favourite new physics model with LHC data, Comput. Phys. Commun. 187 (2015) 227 [arXiv: 1312.2591] [INSPIRE].

[34] DELPHES 3 collaboration, J. de Favereau et al., DELPHES 3, a modular framework for fast simulation of a generic collider experiment, JHEP 02 (2014) 057 [arXiv:1307.6346] [INSPIRE].

[35] M. Cacciari, G.P. Salam and G. Soyez, FastJet user manual, Eur. Phys. J. C 72 (2012) 1896 [arXiv: 1111.6097] [INSPIRE].

[36] M. Cacciari and G.P. Salam, Dispelling the $N^{3}$ myth for the $k_{t}$ jet-finder, Phys. Lett. B 641 (2006) 57 [hep-ph/0512210] [INSPIRE].

[37] M. Cacciari, G.P. Salam and G. Soyez, The anti- $k_{t}$ jet clustering algorithm, JHEP 04 (2008) 063 [arXiv: 0802.1189] [INSPIRE].

[38] ATLAS collaboration, Search for direct top-squark pair production in final states with two leptons in pp collisions at $\sqrt{s}=8 \mathrm{TeV}$ with the ATLAS detector, JHEP 06 (2014) 124 [arXiv: 1403.4853] [INSPIRE].

[39] ATLAS collaboration, Search for strongly produced supersymmetric particles in decays with two leptons at $\sqrt{s}=8 \mathrm{TeV}$, ATLAS-CONF-2013-089 (2013).

[40] ATLAS collaboration, Search for direct-slepton and direct-chargino production in final states with two opposite-sign leptons, missing transverse momentum and no jets in $20 \mathrm{fb}^{-1}$ of $\mathrm{pp}$ collisions at $\sqrt{s}=8 \mathrm{TeV}$ with the ATLAS detector, ATLAS-CONF-2013-049 (2013).

[41] ATLAS collaboration, Search for squarks and gluinos with the ATLAS detector in final states with jets and missing transverse momentum and $20.3 \mathrm{fb}^{-1}$ of $\sqrt{s}=8 \mathrm{TeV}$ proton-proton collision data, ATLAS-CONF-2013-047 (2013).

[42] ATLAS collaboration, Search for direct production of charginos and neutralinos in events with three leptons and missing transverse momentum in $21 \mathrm{fb}^{-1}$ of pp collisions at $\sqrt{s}=8 \mathrm{TeV}$ with the ATLAS detector, ATLAS-CONF-2013-035 (2013).

[43] ATLAS collaboration, Search for direct production of the top squark in the all-hadronic $t \bar{t}+$ Etmiss final state in $21 \mathrm{fb}^{-1}$ of pp collisions at $\sqrt{s}=8 \mathrm{TeV}$ with the ATLAS detector, ATLAS-CONF-2013-024 (2013).

[44] ATLAS collaboration, Search for new phenomena in monojet plus missing transverse momentum final states using $10 \mathrm{fb}^{-1}$ of pp collisions at $\sqrt{\mathrm{s}}=8 \mathrm{TeV}$ with the ATLAS detector at the LHC, ATLAS-CONF-2012-147 (2012). 
[45] ATLAS collaboration, Search for supersymmetry at $\sqrt{s}=8 \mathrm{TeV}$ in final states with jets, missing transverse momentum and one isolated lepton, ATLAS-CONF-2012-104 (2012).

[46] ATLAS collaboration, Search for new phenomena in final states with an energetic jet and large missing transverse momentum in pp collisions at $\sqrt{s}=8 \mathrm{TeV}$ with the ATLAS detector, Eur. Phys. J. C 75 (2015) 299 [arXiv:1502.01518] [INSPIRE].

[47] ATLAS collaboration, ATLAS Run 1 searches for direct pair production of third-generation squarks at the Large Hadron Collider, Eur. Phys. J. C 75 (2015) 510 [arXiv:1506.08616] [INSPIRE].

[48] ATLAS collaboration, Search for new phenomena in events with a photon and missing transverse momentum in pp collisions at $\sqrt{s}=8 \mathrm{TeV}$ with the ATLAS detector, Phys. Rev. D 91 (2015) 012008 [arXiv:1411.1559] [InSPIRE].

[49] ATLAS collaboration, Search for pair-produced third-generation squarks decaying via charm quarks or in compressed supersymmetric scenarios in pp collisions at $\sqrt{s}=8 \mathrm{TeV}$ with the ATLAS detector, Phys. Rev. D 90 (2014) 052008 [arXiv: 1407.0608] [InSPIRE].

[50] ATLAS collaboration, Search for top squark pair production in final states with one isolated lepton, jets and missing transverse momentum in $\sqrt{s}=8 \mathrm{TeV}$ pp collisions with the ATLAS detector, JHEP 11 (2014) 118 [arXiv:1407.0583] [INSPIRE].

[51] ATLAS collaboration, Search for squarks and gluinos with the ATLAS detector in final states with jets and missing transverse momentum using $\sqrt{s}=8$ TeV proton-proton collision data, JHEP 09 (2014) 176 [arXiv:1405.7875] [INSPIRE].

[52] ATLAS collaboration, Search for supersymmetry at $\sqrt{s}=8$ TeV in final states with jets and two same-sign leptons or three leptons with the ATLAS detector, JHEP 06 (2014) 035 [arXiv: 1404.2500] [INSPIRE].

[53] ATLAS collaboration, Search for direct top squark pair production in events with a $Z$ boson, $b$-jets and missing transverse momentum in $\sqrt{s}=8 \mathrm{TeV}$ pp collisions with the ATLAS detector, Eur. Phys. J. C 74 (2014) 2883 [arXiv:1403.5222] [INSPIRE].

[54] ATLAS collaboration, Search for direct production of charginos and neutralinos in events with three leptons and missing transverse momentum in $\sqrt{s}=8 \mathrm{TeV}$ pp collisions with the ATLAS detector, JHEP 04 (2014) 169 [arXiv: 1402.7029] [INSPIRE].

[55] ATLAS collaboration, Search for direct third-generation squark pair production in final states with missing transverse momentum and two b-jets in $\sqrt{s}=8 \mathrm{TeV}$ pp collisions with the ATLAS detector, JHEP 10 (2013) 189 [arXiv:1308.2631] [INSPIRE].

[56] ATLAS collaboration, Search for new phenomena in final states with large jet multiplicities and missing transverse momentum at $\sqrt{s}=8$ TeV proton-proton collisions using the ATLAS experiment, JHEP 10 (2013) 130 [Erratum ibid. 01 (2014) 109] [arXiv:1308.1841] [INSPIRE].

[57] ATLAS collaboration, Search for supersymmetry in events containing a same-flavour opposite-sign dilepton pair, jets and large missing transverse momentum in $\sqrt{s}=8 \mathrm{TeV} p p$ collisions with the ATLAS detector, Eur. Phys. J. C 75 (2015) 318 [arXiv:1503.03290] [INSPIRE].

[58] ATLAS collaboration, Search for strong production of supersymmetric particles in final states with missing transverse momentum and at least three b-jets using $20.1 \mathrm{fb}^{-1}$ of pp collisions at $\sqrt{s}=8$ TeV with the ATLAS Detector, ATLAS-CONF-2013-061 (2013).

[59] C. Kounnas, A.B. Lahanas, D.V. Nanopoulos and M. Quirós, Low-energy behavior of realistic locally supersymmetric grand unified theories, Nucl. Phys. B 236 (1984) 438 [InSPIRE]. 
[60] J.P. Derendinger and C.A. Savoy, Quantum effects and $\mathrm{SU}(2) \times \mathrm{U}(1)$ breaking in supergravity gauge theories, Nucl. Phys. B 237 (1984) 307 [INSPIRE].

[61] J.M. Frere, D.R.T. Jones and S. Raby, Fermion masses and induction of the weak scale by supergravity, Nucl. Phys. B 222 (1983) 11 [INSPIRE].

[62] R. Rattazzi and U. Sarid, Large $\tan \beta$ in gauge mediated SUSY breaking models, Nucl. Phys. B 501 (1997) 297 [hep-ph/9612464] [INSPIRE].

[63] J. Hisano and S. Sugiyama, Charge-breaking constraints on left-right mixing of stau's, Phys. Lett. B 696 (2011) 92 [Erratum ibid. B 719 (2013) 472] [arXiv: 1011.0260] [INSPIRE].

[64] M. Carena, S. Gori, I. Low, N.R. Shah and C.E.M. Wagner, Vacuum stability and higgs diphoton decays in the MSSM, JHEP 02 (2013) 114 [arXiv:1211.6136] [INSPIRE].

[65] T. Kitahara and T. Yoshinaga, Stau with large mass difference and enhancement of the Higgs to diphoton decay rate in the MSSM, JHEP 05 (2013) 035 [arXiv:1303.0461] [INSPIRE].

[66] J.E. Camargo-Molina, B. O'Leary, W. Porod and F. Staub, Stability of the CMSSM against sfermion VEVs, JHEP 12 (2013) 103 [arXiv:1309.7212] [INSPIRE].

[67] D. Chowdhury, R.M. Godbole, K.A. Mohan and S.K. Vempati, Charge and color breaking constraints in MSSM after the Higgs discovery at LHC, JHEP 02 (2014) 110 [arXiv: 1310.1932] [INSPIRE].

[68] J.E. Camargo-Molina, B. O'Leary, W. Porod and F. Staub, Vevacious: a tool for finding the global minima of one-loop effective potentials with many scalars, Eur. Phys. J. C $\mathbf{7 3}$ (2013) 2588 [arXiv: 1307.1477] [INSPIRE].

[69] T. Moroi, H. Murayama and M. Yamaguchi, Cosmological constraints on the light stable gravitino, Phys. Lett. B 303 (1993) 289 [INSPIRE].

[70] M. Pospelov, Particle physics catalysis of thermal Big Bang nucleosynthesis, Phys. Rev. Lett. 98 (2007) 231301 [hep-ph/0605215] [INSPIRE].

[71] M. Kawasaki, K. Kohri, T. Moroi and A. Yotsuyanagi, Big-Bang nucleosynthesis and gravitino, Phys. Rev. D 78 (2008) 065011 [arXiv:0804.3745] [INSPIRE].

[72] J. Kersten and K. Schmidt-Hoberg, The gravitino-stau scenario after catalyzed BBN, JCAP 01 (2008) 011 [arXiv:0710.4528] [INSPIRE]. 